\title{
Parallel versus Hierarchical Fusion of Extended Fingerprint Features
}

\author{
Qijun Zhao, Feng Liu, Lei Zhang, David Zhang \\ Biometrics Research Centre, Department of Computing, The Hong Kong Polytechnic University \\ \{csqjzhao, csfliu, cslzhang, csdzhang\}@comp.polyu.edu.hk
}

\begin{abstract}
Extended fingerprint features such as pores, dots and incipient ridges have been increasingly attracting attention from researchers and engineers working on automatic fingerprint recognition systems. A variety of methods have been proposed to combine these features with the traditional minutiae features. This paper comparatively analyses the parallel and hierarchical fusion approaches on a high resolution fingerprint image dataset. Based on the results, a novel and more effective hierarchical approach is presented for combining minutiae, pores, dots and incipient ridges.
\end{abstract}

\section{Introduction}

Traditional automated fingerprint recognition systems (AFRS) are mainly based on the minutiae features, i.e. ridge endings and bifurcations [1-2]. In recent years, increasing attention has been paid to extended fingerprint features such as pores, dots and incipient ridges (see Fig. 1) for the purpose of further enhancing the fingerprint recognition accuracy. While extended features are routinely used by experts in manual latent fingerprint matching [3-4], they have been exploited in AFRS only recently thanks to the advent of high quality fingerprint sensors [5]. They have been proven to be able to improve the accuracy of AFRS when combined with minutiae [6-11].

A number of methods, including parallel and hierarchical fusion approaches, have been proposed for fusing minutiae and extended features. In [7] and [9], for example, the authors combined minutiae with pores and dots and incipient ridges by summing up their match scores, which is parallel fusion. In [5], on the other hand, pores were compared first and if they could not be well matched, the input fingerprint was rejected directly; otherwise, minutiae were further compared. This is hierarchical fusion, which was also used by [8] and [10]. The method of [8] proceeds

\footnotetext{
This research is partially supported by the HK PolyU internal
} research grant G-YH54. from level-1 features to level-3 features, and directly rejects the input fingerprint if any of the features are found to be unmatched. In [10], level-2 (i.e. minutiae) features were also compared before level-3 (i.e. Pores, ridge contours, and edgeoscopic) features, but the input fingerprint was directly accepted (rather than rejected) if the level-2 features were matched well and the level-3 features would not be further compared.

From above discussion, we can see that given the various approaches in the literature of fusing extended features and minutiae, an extensive study on them is highly necessary to investigate how extended fingerprint features can be better utilized by AFRS. Toward the end, this paper will comparatively analyze the parallel and hierarchical fusion approaches by using a high resolution fingerprint image dataset. Some basic problems in designing hierarchical fusion approaches will be discussed for fingerprint features. A novel hierarchical fusion method is then presented to combine minutiae, pores, dots and incipient ridges, and it is shown to be more effective than existing approaches.

The rest of the paper is organized as follows. Section 2 discusses different parallel and hierarchical fusion approaches, including both existing and the newly proposed ones. Section 3 reports and analyzes the evaluation results. Section 4 concludes the paper.

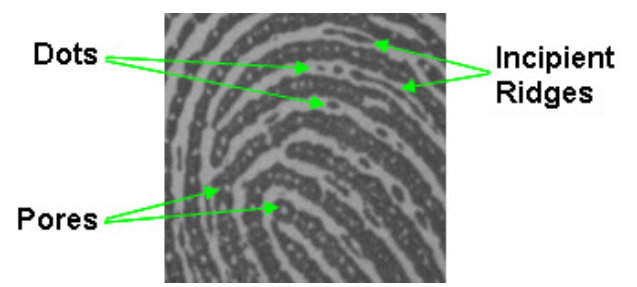

Figure 1. Example extended fingerprint features: pores, dots and incipient ridges.

\section{Fusion Approaches}

\subsection{Parallel Fusion}


Generally, the fusion in a biometric system can be fulfilled at four different levels, i.e. sensor level, feature level, score level, and decision level [12]. This study focuses on the score level fusion of minutiae and extended fingerprint features, which is most widely used in fingerprint recognition. In parallel fusion approaches, different fingerprint features are matched separately and simultaneously, each defining a matcher. Score normalization is then applied to change the location and scale parameters of the match score distributions at the outputs of the individual matchers so that the match scores of different matchers are transformed into a common domain [12]. The min$\max (\mathrm{MMN})$ and z-score normalization techniques [12] are considered in our experiments. When applying zscore normalization, the mean and standard deviation of match score distribution are estimated in two different ways. One uses the mean and standard deviation of genuine match scores (ZNG), and the other uses those of imposter match scores (ZNI). After normalization, the match scores of individual matchers are combined to form one single final score for the input fingerprint by using the min (MIN), max (MAX), and simple sum (SSUM) rules. The MIN and MAX rules respectively pick as the final score the minimum and maximum of the match scores of all individual matchers, whereas the SSUM rule takes the summation of the match scores as the final score [12].

\subsection{Hierarchical Fusion}

Hierarchical fusion, in contrast to parallel fusion, launches the matchers in serial. The recognition process can stop at any matcher if the matcher can already make a decision with high confidence; otherwise, it will proceed to the matcher at the next layer until a decision can be made. Two basic problems are involved in designing hierarchical fusion approaches: 1) in which order should the matchers/features be applied and 2) in what manner should the features be used? For example, one kind of features can be used in a positive manner, i.e. if its match score is above a given threshold, then the input fingerprint is directly accepted as a genuine; or in a negative manner, i.e. if its match score is not above the threshold, then the input fingerprint is directly rejected as an imposter. Different orders and both manners have been used in previous work [5-10]. However, so far, no comparative study has been reported regarding these approaches, and it is still unclear which approach is better for combining minutiae and extended fingerprint features.

In this paper, we will consider two different orders: from minutiae to pores to dots and incipient ridges (denoted as MPD), and inversely (denoted as DPM).
In each order, we consider using the features in both positive (denoted as $\mathrm{P}$ ) and negative (denoted as $\mathrm{N}$ ) manners. Thus, four different hierarchical fusion approaches are studied here, MPD_P, MPD_N, DPM_P, and DPM_N, among which DPM_P is for the first time proposed in the literature. Fig. 2 shows the flowcharts of MPD_N and DPM_P as examples. In the figure, $s_{m}, s_{p}$, and $s_{d}$ denote respectively the match scores of minutiae, pores, and dots and incipient ridges. With respect to the associated thresholds, $t_{m}, t_{p}$, and $t_{d}$, we chose them in the experiments according to the manner in which the features were used. Specifically, if a feature is used in a positive manner on prior layers of the fusion hierarchy, we selected the threshold for it as the minimum of the thresholds which produce the minimum false acceptance rate (FAR). This is because such threshold can give the lowest false rejection rate (FRR) among all the thresholds that correspond to the minimum FAR. Similarly, for a feature negatively used, we selected its threshold as the maximum of the thresholds which lead to the minimum FRR.

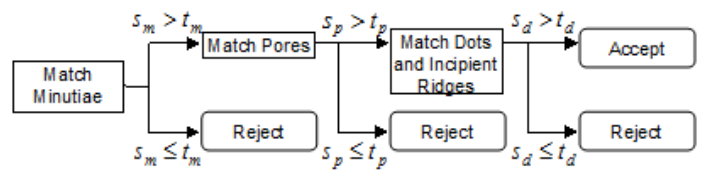

(a)

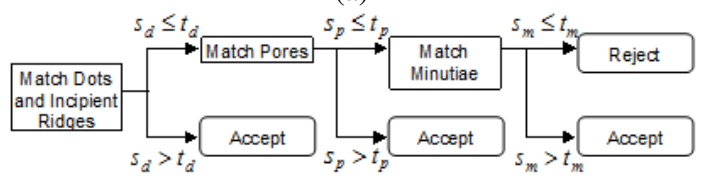

(b)

Figure 2. The flowcharts of two example hierarchical fusion approaches: (a) MPD_N and (b) DPM_P.

\section{Evaluation and Analysis}

\subsection{Dataset and Algorithms}

Due to the lack of large database of high resolution fingerprint images in public domain, a set of high resolution ( 1200dpi) fingerprint images were collected by using our custom-built acquisition device to evaluate different fusion approaches. The dataset consists of 1,480 fingerprint images from 148 fingers, each having five images captured in each of two sessions (about two weeks apart). The images have a spatial size of $640 \times 480$ pixels.

The minutiae were extracted from the fingerprint images and matched by using the method in [13]. The pores on the images were extracted by using an improved method of [14], and matched by using the method in [15]. The dots and incipient ridges were extracted and matched by using methods similar to 
those in [14] and [15]. Note that the minutiae match score between two fingerprints is defined as the percentage of the matched minutiae among the complete set of minutiae on the two fingerprints. The pore match score between them is defined as the number of finally matched pores on them. The dots and incipient ridges are matched together by representing both of them with the coordinates of their centers, and the match score of dots and incipient ridges on two fingerprints is also defined as the number of matched dots and incipient ridges on them.

With the above feature extraction and matching methods, the following matches were conducted for each individual feature. 1) Genuine matches: each of the fingerprint images in the second session was matched with all the fingerprint images of the same finger in the first session, resulting in 3,700 genuine match scores. 2) Imposter matches: the first fingerprint image of each finger in the second session was matched with the first fingerprint images of all the other fingers in the first session, resulting in 21,756 imposter match scores. Based on these match scores, the equal error rates (EER) and the receiver operating characteristic (ROC) curves of different parallel fusion and hierarchical fusion methods were calculated. In the remaining part of this section, we report and analyze the obtained results.

\subsection{Fusion Results}

The EERs of different parallel fusion approaches are listed in Table 1. The best result among these parallel fusion approaches is obtained by MMN+SSUM, and the corresponding ROC curve (denoted by MPD_MMN_SSUM) is plotted in Fig. 3 together with the ROC curves of different hierarchical fusion approaches. For comparison, the ROC curves of individual features are also displayed in Fig. 3.

Table 1. The equal error rates of different parallel fusion methods.

\begin{tabular}{c|c|c|c}
\hline & MIN & MAX & SSUM \\
\hline MMN & $13.54 \%$ & $0.71 \%$ & $\mathbf{0 . 6 5 \%}$ \\
\hline ZNG & $44.61 \%$ & $13.92 \%$ & $0.92 \%$ \\
\hline ZNI & $19.48 \%$ & $16.22 \%$ & $0.8 \%$ \\
\hline
\end{tabular}

\subsection{Analysis}

The above results show that the best EERs obtained by parallel and hierarchical fusion are respectively $0.65 \%$ and $0.508 \%$. This supports the conclusion of previous studies [7-8] that hierarchical fusion is better than parallel fusion in combing fingerprint features. However, our results also show that in order to get the best performance by using hierarchical fusion, the order and manner of using fingerprint features should be carefully considered; if the features are not properly utilized, hierarchical fusion can even work worse than parallel fusion.

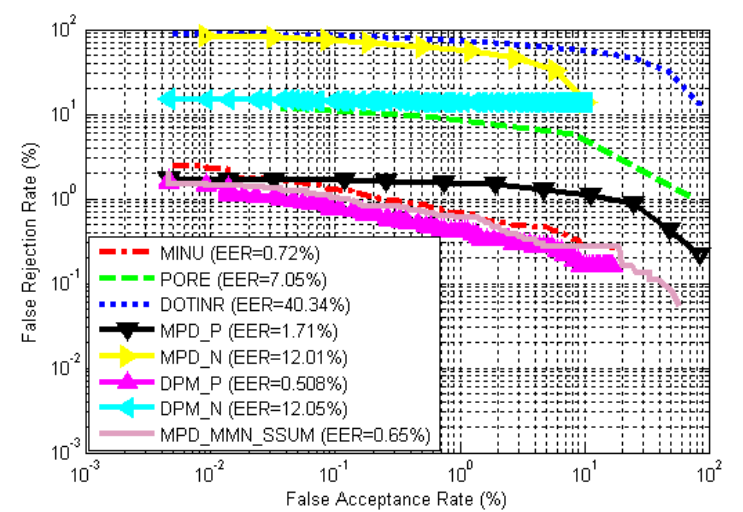

Figure 3. The ROC curves of individual features and different parallel and hierarchical fusion approaches.

Among different hierarchical fusion approaches, the positive manner overwhelms the negative one in all cases. When the features are used in a negative manner, the performance after fusion becomes even worse than that achieved by individual features. In order to better understand why the positive manner is better, we show in Fig. 4 the match score distributions of the individual features, i.e. minutiae, pores, dots and incipient ridges. We also calculated the following statistics on these match scores: the minimum (Min), maximum (Max), mean (Mean), and standard deviation (Std) of the genuine and imposter match scores. The results are presented in Table 2.

Table 2. The (genuine, imposter) match score statistics of individual features.

\begin{tabular}{c|c|c|c|c}
\hline & Min & Max & Mean & Std \\
\hline \multirow{2}{*}{ MINU } & $(0,0)$ & $\begin{array}{c}(0.99, \\
0.23)\end{array}$ & $\begin{array}{c}(0.61, \\
0.02)\end{array}$ & $\begin{array}{c}(0.179, \\
0.036)\end{array}$ \\
\hline \multirow{2}{*}{ PORE } & $(5,0)$ & $(396,9)$ & $(68,6)$ & $\begin{array}{c}(63.5, \\
0.6)\end{array}$ \\
\hline DOTINR & $(0,0)$ & $(65,14)$ & $(5.9,2.6)$ & $\begin{array}{c}(6.96, \\
1.63)\end{array}$ \\
\hline
\end{tabular}

According to Fig. 4 and Table 2, the genuine and imposter match scores of all of the considered features have overlap in the lower part of the range of match scores. This means that some pairs of genuine fingerprints would also fall into the cluster obtaining low match scores. Consequently, if we directly reject an input fingerprint just because its match score of certain feature is small as what we have done on the prior layers of hierarchical fusion in negative manner, it will be very possible for us to commit a false rejection. On the other hand, because there is no 
overlap between genuine and imposter match scores on the higher part of the range of scores, we can accept with high confidence the input fingerprint which gains a high match score.

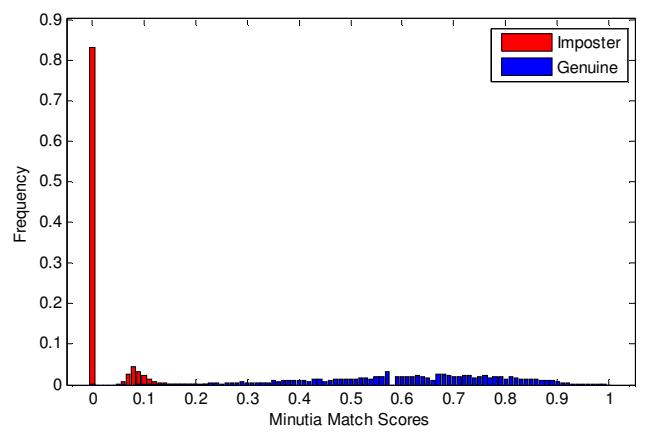

(a)

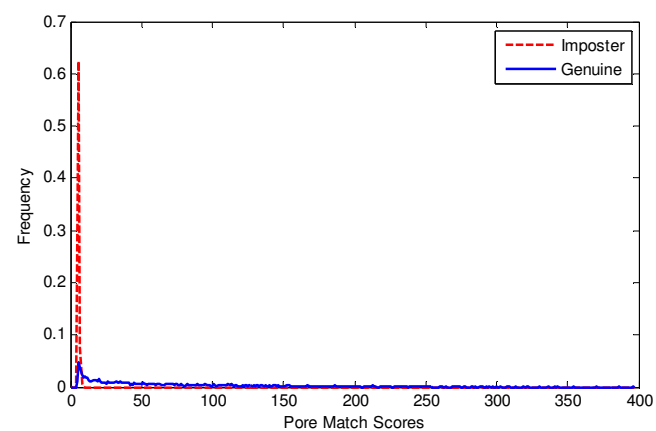

(b)

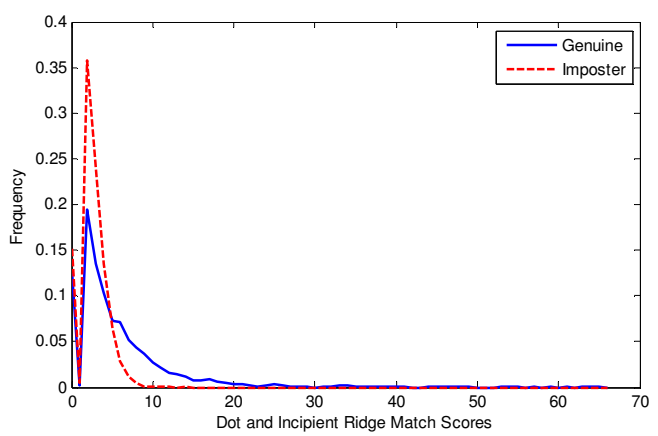

(c)

Figure 4. The match score distributions of (a) minutiae, (b) pores, and (c) dots and incipient ridges.

Furthermore, when the positive manner is taken, the fusion from DOTINR to PORE to MINU outperforms the fusion using an inverse order. This is interesting considering that in most previous studies $[8$, 10], the fingerprint features on level-2 were compared before those on level-3. The results here, however, show that using the extended fingerprint features in a positive manner and on the prior layers of the hierarchical fusion system can benefit more the overall accuracy of AFRS.

\section{Conclusions}

This paper comparatively studied parallel and hierarchical fusion approaches for combining minutiae and extended fingerprint features. The results showed the advantages of hierarchical fusion over parallel fusion, and demonstrated the importance of fusion order and manner in designing hierarchical fusion approaches. A more effective hierarchical fusion approach has also been presented in the study.

\section{References}

[1] N. Ratha and R. Bolle. Automatic Fingerprint Recognition Systems. Springer, New York, 2004.

[2] D. Maltoni, D. Maio, A. K. Jain, and S. Prabhakar. Handbook of Fingerprint Recognition. $2^{\text {nd }}$ Edition. Springer, New York, 2009.

[3] D. R. Ashbaugh. Quantitative-Qualitative Friction Ridge Analysis: An Introduction to Basic and Advanced Ridgeology. CRC Press LLC, 1999.

[4] CDEFFS. Data Format for the Interchange of Extended Fingerprint and Palmprint Features, Version 0.4. 2009.

[5] J. D. Stosz and L. A. Alyea. Automated System for Fingerprint Authentication Using Pores and Ridge Structure. SPIE, 2277: 210-223, 1994.

[6] K. Kryszczuk, P. Morier, and A. Drygajlo. Study of the Distinctiveness of Level 2 and Level 3 Features in Fragmentary Fingerprint Comparison. BioAW2004, LNCS 3087, 124-133, 2004.

[7] A. K. Jain, Y. Chen, and M. Demirkus. Pores and Ridges: Fingerprint Matching Using Level 3 Features. ICPR'06, 4: 477-480, 2006.

[8] A. K. Jain, Y. Chen, and M. Demirkus. Pores and Ridges: High-Resolution Fingerprint Matching Using Level 3 Features. IEEE Trans. PAMI, 29(1): 15-27, 2007.

[9] Y. Chen and A. K. Jain. Dots and Incipients: Extended Features for Partial Fingerprint Matching. Presented at Biometric Symposium, Baltimore, 2007.

[10] International Biometric Group. Analysis of Level 3 Features at High Resolutions, Phase II - Final Report. 2008.

[11] M. Vasta, R. Singh, A. Noore, and S. K. Singh. Combining Pores and Ridges with Minutiae for Improved Fingerprint Verification. Signal Processing, 89: 2676-2685, 2009.

[12] A. Ross, K. Nandakumar, and A. K. Jain. Handbook of Multibiometrics. Springer, 2006.

[13] J. Feng. Combining Minutiae Descriptors for Fingerprint Matching. Pattern Recognition, 41: 342-352, 2008.

[14] Q. Zhao, L. Zhang, D. Zhang, N. Luo, and J. Bao. Adaptive Pore Model for Fingerprint Pore Extraction. ICPR'08, 2008.

[15] Q. Zhao, L. Zhang, D. Zhang, and N. Luo. Direct Pore Matching for Fingerprint Recognition. ICB'09: 597-606, 2009. 